\title{
Fish assemblages of European tidal marshes: a comparison based on species, families and functional guilds
}

\author{
S. Mathieson ${ }^{1, *}$, A. Cattrijsse ${ }^{2,3}$, M. J. Costa ${ }^{4}$, P. Drake ${ }^{5}$, M. Elliott ${ }^{6}$, J. Gardner $^{6}$, \\ J. Marchand ${ }^{7, \mathrm{I}}$ \\ ${ }^{1}$ Scottish Environment Protection Agency, Erskine Court, The Castle Business Park, Stirling FK9 4TR, United Kingdom \\ ${ }^{2}$ Department of Biology, Marine Biology Section, University of Gent, K.L. Ledeganckstraat 35, 9000 Gent, Belgium \\ ${ }^{3}$ Institute for Marine Scientific Research (IZWO), Victorialaan 3, 8400 Oostende, Belgium \\ ${ }^{4}$ Universidade de Lisboa, Departmento de Zoologia, Fac. de Ciencias, Bloco C2, 3e Piso, Campo Grande, 1700 Lisboa, Portugal \\ ${ }^{5}$ Instituto de Ciencias Marinas de Andalucia, Poligono Rio San Pedro, s/n 11510 Puerto Real, Cadiz, Spain \\ ${ }^{6}$ Institute of Estuarine and Coastal Studies, University of Hull, Hull HU6 7RX, United Kingdom \\ ${ }^{7}$ Université de Nantes, Laboratoire de Biologie Marine, 2, Rue de la Houssiniere, 44322 Nantes Cedex 3, France
}

\begin{abstract}
In considering the use of estuarine habitats by fish assemblages, alternative views of the assemblage are increasingly being explored, based on functional rather than taxonomic aspects. The allocation of all taxa to a number of functional guilds allows a description of fish assemblages in terms of vertical zonation, habitat preferences, including the substratum preference of benthic/demersal species, and dietary preferences. This paper presents the first comparison of the structure of the fish assemblages in the tidal marshes of 6 European estuaries (Bay of Cadiz, Spain; Mira, Portugal; Loire, France; Westerschelde, The Netherlands; Humber, UK; Forth, UK). Cluster analysis was used to compare the assemblages recorded in 29 tidal marsh and associated estuary reference sites. Distinct differences between estuaries were apparent in comparing the taxonomic structure (species, family) of the assemblages, indicating a division between assemblages of northern and southern Atlantic European tidal marshes. The use of ecological guilds successfully separated marsh assemblages dominated by freshwater adventitious/diadromous taxa (Loire) from those dominated by marine juvenile taxa (Mira, Forth). Comparisons of vertical distribution guilds indicated a major division between pelagic-dominated (Forth, upper Loire) and benthic-dominated (Cadiz, Westerschelde, Mira, Loire) assemblages. This division was also partly reflected by the dietary preference guilds, with a strong separation between assemblages dominated by planktivores (Forth) and those in which most taxa are either invertebrate feeders (Humber, Cadiz, Westerschelde) or invertebrate/fish feeders (most Mira sites, lower Loire). The very low representation of a primarily piscivorous guild in all sites (in contrast to the fish assemblages of the main estuaries) supports the view that European tidal marshes contain fish assemblages with high proportions of juvenile individuals and may provide significant refugia for life stages vulnerable to predation. Although the comparability of datasets is limited by differences in sampling regimes and methodologies, the use of functional guilds, reflecting different aspects of the use of the marshes, reveals that functional similarities exist between the fish assemblages of European tidal marshes, despite considerable taxonomic, physical and chemical differences between the sites. Further modifications to the use of functional guilds are discussed, and recommendations made to refine their value for studying fish assemblages in tidal marsh environments.
\end{abstract}

KEY WORDS: Europe $\cdot$ Tidal marshes $\cdot$ Fish assemblages $\cdot$ Functional guilds

Resale or republication not permitted without written consent of the publisher

\section{INTRODUCTION}

Estuaries have long been recognised as areas which naturally support high densities and production levels of fish, as permanent residents, during migration, or

(C) Inter-Research 2000 during specific life stages, particularly as juveniles (e.g. McHugh 1967, Haedrich 1983, Blaber 1991). Despite the significance of estuaries as habitats for life stages of fish species of commercial importance (e.g.

\footnotetext{
*E-mail: scot.mathieson@sepa.org.uk

${ }^{\ddagger}$ Deceased
} 
Naismith \& Knights 1988, Elliott et al. 1990) or which support fish species of international conservation value (e.g. Maitland 1974, Aprahamian 1988), anthropogenic pressures continue to threaten the integrity and quality of estuaries world-wide (McLusky 1989, GESAMP 1997). An understanding of the use of estuaries by fish and the roles and importance to fish assemblages of sub-habitats within the estuary is, therefore, required by those responsible for management decisions affecting estuaries.

The use of functional guilds has been proposed as an alternative to the analysis of traditional taxonomic divisions of fish assemblages and to provide a better understanding of the structure and functioning of the assemblages of fish occurring in estuaries. Elliott \& Dewailly (1995) recently proposed 29 functional guilds for the classification of estuarine fish, covering ecological types, vertical zonation, substratum and diet preferences, and reproductive strategies. This built on earlier attempts using an ecological guild system (McHugh 1967, Potter et al. 1990, 1993) or dietary preferences (Morton et al. 1987). Similar approaches have been attempted for rivers, to generate several taxonomic and functional measures to be used as 'metrics' to establish a fish-based index of biological integrity for rivers (Karr 1981, Didier \& Kestemont 1996, Hugueny et al. 1996).

A fish-based index of biological integrity, based on similar taxonomic and guild categories, has also recently been applied successfully to estuarine systems in Massachusetts, USA (Deegan et al. 1997). The purpose of Elliott \& Dewailly (1995), however, was to provide alternative ways of describing and exploring the use of estuaries by fish. Classification analysis of fish assemblage data from 17 European estuaries, based on the 29 guilds, provided insights into fish use of the individual estuaries not apparent from a conventional taxonomic (species, family) analysis (Elliott \& Dewailly 1995). A limitation of this analysis of wholeestuary assemblages identified by the authors was a failure to consider the use made by fish of individual sub-habitats within the estuaries. This was a result of the diverse nature of the individual studies combined to form the data matrix for the study, which meant that the allocation of individual species' records to subhabitats within the estuaries was not feasible.

The role of tidal marshes, which are vegetated and regularly tidally flooded habitats found in intertidal zones in temperate regions of the world, as habitats for estuarine fish and other nekton was reviewed in detail by Kneib (1997). He considered that the overall contribution of marsh nekton to estuarine nekton assemblages has generally been underestimated. He also indicated that few published data are available to describe European marsh nekton assemblages, confirming a similar earlier statement by Cattrijsse et al. (1994). These authors also reported that, in contrast to North American Atlantic salt marshes, where vegetation occurs down to the mean tidal level (MTL) and extensive use is made by nekton of the vegetated intertidal area, European marshes are bordered on the down-shore side by the mean high water neap (MHWN) tide level. In consequence, as well as being a much more marginal estuarine habitat in Europe than in North America, the intertidal habitats of European marshes, unlike those of American marshes, are usually flooded for only short periods during relatively high tides. Nekton making tidal migrations into the intertidal marsh are, therefore, nearly always confined to the intertidal creeks.

The aim of the present study is to develop further the approach of Elliott \& Dewailly (1995), comparing here the functional aspects of the fish assemblages using tidal marshes in 6 European estuaries. The study compares taxonomic and functional aspects of the assemblages and considers whether a typical European tidal marsh fish assemblage may be identified, either taxonomically or functionally distinct from the fish assemblages of the main estuary.

\section{MATERIAL AND METHODS}

Sources of data. A number of published and previously unpublished datasets were combined for this study. Analysis based on only abundance/density data was unavoidable as not all component studies collected data on biomass. The source of each dataset is described below. Published data were available for the Westerschelde estuary (The Netherlands) and the Bay of Cadiz (Spain) and unpublished data for the Westerschelde, the Forth estuary (Scotland), the Humber estuary (England), the Loire estuary (France) and the Mira estuary (Portugal) (Fig. 1).

Study sites and sampling details. Data were available for a total of 29 individual tidal marsh and related estuary channel sites in the 6 estuaries. Locations, characteristics and sampling details for tidal marsh sites and sampling methods for estuarine reference sites in the 6 estuaries are described in Table 1 and below.

Forth estuary (Latitude: $56^{\circ} \mathbf{1 0}^{\prime} \mathbf{N}$ ): Intertidal creeks are only present in a narrow strip of marshes upstream of Kincardine (Burd 1989). The Kincardine marsh is situated in the mesohaline part of the estuary and is therefore the most saline strip of marsh in the estuary as marsh sites downstream have largely been lost to development land claim (Mathieson pers. obs.). Narrow, relatively short and shallow, linear branched creeks run through Puccinellia maritima-dominated stands (Proctor et al. 1982). An 8 mm meshed block net sampled the ebb current in the creek (site code: F1) 


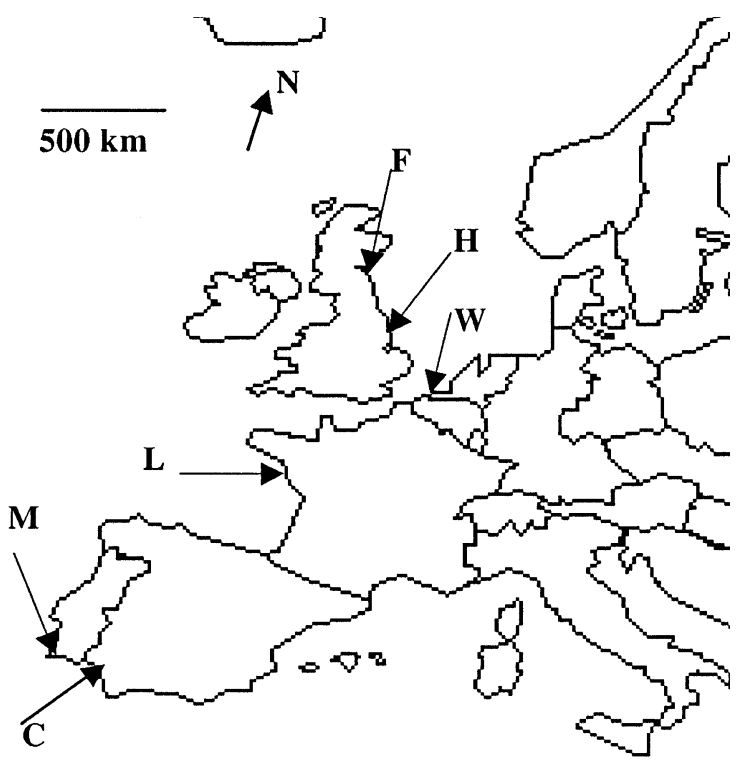

Fig. 1. Location of the 6 estuaries included in the study.

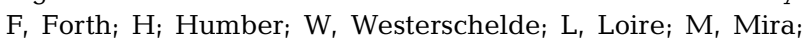
C, Bay of Cadiz

while beam-trawl samples were collected ( $8 \mathrm{~mm}$ mesh) in the subtidal area in front and upstream of the marsh (F2 and F3 respectively: R. Park, Scottish Environment Protection Agency, unpubl. data).

Humber estuary (Latitude: $53^{\circ} 40^{\prime} N$ ): The Welwick marsh occupies a sheltered position on the north bank of the Humber and is the largest saltmarsh in the estuary, with a well-developed creek system and a clear zonation in the vegetation. The saltmarsh is bounded at its upper level by flood defence walls and by relatively hard-packed mud at the lower level. Spartina spp. (probably anglica) dominates the lower marsh, forming a wide sward with lower abundance of Salicornia europea and Suaeda maritima. Puccinellia maritima, Elymus athericus and Halimione maritima are abundant in the higher marsh. In the upper area, the main species to co-exist with $P$. maritima is the sea-aster Aster tripolium. The creeks are up to $1 \mathrm{~m}$ deep especially at the entrance to the marsh and thus, in places, retain water even at low tide. Creeks were sampled with fyke nets and additionally with a block net identical to the one used in the Forth (F1). These data have not been reported before. Data on the subtidal fish assemblages and environment have been published by Marshall \& Elliott (1996). The sampling methodology of the subtidal sites (H2 and $\mathrm{H} 3$ ) is also the same as that applied in the Forth subtidal reference sites (F2 and F3).

Westerschelde estuary (Latitude: $51^{\circ} 20^{\prime} N$ ): Samples were collected in the creeks of 2 estuarine fringing marshes. A stow net with a $1 \mathrm{~mm}$ mesh was used in these marsh creeks. Saeftinghe (W1), which is the largest European estuarine marsh, is situated in the oligohaline zone of the estuary. Just a few kilometres upstream, the estuary is anoxic year round, and during periods of high runoff the concentration of dissolved oxygen in the creek can be low. Six plants dominate the vegetation of the sampling site with Puccinellia maritima as the most abundant. Saeftinghe is the only marsh in the Westerschelde with a distinct zonation in the vegetation. The nekton assemblage of this intertidal creek was described by Cattrijsse et al. (1994).

Data from the smaller mesohaline marsh of Waarde (W2) have not been published previously. Unlike Saeftinghe, this marsh is not grazed, has only 1 major creek, and an extensive system of tidal flats and shallow gullies stretches out in front of the marsh. The water here is always well oxygenated. As with Saeftinghe, the vegetation has no real dominant species but Puccinellia maritima is the most abundant species. Other important species include Elymus athericus, Triglochin maritima and Atriplex hastata. On Saeftinghe, Scirpus maritimus and Phragmites communis are also important members of the flora. The subtidal samples were collected in the main channel of the estuary near Kruiningen, close to the marsh of Waarde, using a $3 \mathrm{~m}$ beam trawl with a $5 \mathrm{~mm}$ meshed cod end, with a $1 \mathrm{~km}$ tow length.

Loire estuary (Latitude: $47^{\circ} 10^{\prime} \mathrm{N}$ ): The study area is an impounded marsh area, situated near Lavau a few kilometres upstream of the estuary mouth. The whole area is cut off from regular tidal influence of the estuary's mesohaline zone by 2 gates at the mouth of the main channel which are operated periodically to control the water level within the marsh system. One intertidal reference site was situated in the small Lavau tributary to the estuary (L1). Along the bank of this shallow channel Scirpus maritimus grows. Another tidal site (L2) was downstream of the first gate, while all other sites were without tidal influence except when the sluice gates were opened. L3 was just upstream of the first gate and L4 was also taken in the channel between the 2 gates (Canal du Syl), $400 \mathrm{~m}$ upstream, while L5 was upstream of the second gate. Sites L6, L7 and L8 were in the main channel of the freshwater marsh (Etier Lavau). Sites L9 and L10 were in a smaller canal tributary of the main channel (Pré Neuf marshes) some $4 \mathrm{~km}$ from the Loire. Along the creek banks of the impounded areas Phragmites communis is abundant. The pastured meadows around the impounded canals have Polypogon monspeliensis as the main plant species.

Several sampling methods were employed in these freshwater habitats: a fyke net ( $5 \mathrm{~mm}$ mesh size) in sites L4 to L10; in L4, additional samples were taken with a fyke net with a $10 \mathrm{~mm}$ mesh; in the remaining sites glass-eel tow nets (a type of hand-drawn pull net) and lift nets were used. All data have not previously been published, other than in an internal report (Marchand \& 
Table 1. Locations, characteristics and sampling details for tidal marsh sites (sites are intertidal unless otherwise indicated) and sampling methods for estuarine reference sites in 6 European estuaries. n/s: not sampled or not recorded

\begin{tabular}{|c|c|c|c|c|c|c|c|}
\hline $\begin{array}{l}\text { Humber, } \\
\text { England }\end{array}$ & $\begin{array}{l}\text { Welwick } \\
\text { (H1) }\end{array}$ & $\begin{array}{l}\text { Estuarine } \\
\text { fringe }\end{array}$ & 32 & $\begin{array}{l}\text { Linear } \\
\text { dendritic }\end{array}$ & Narrow & $\begin{array}{l}\text { Spartina } \\
\text { spp. }\end{array}$ & Mud \\
\hline $\begin{array}{l}\text { Westerschelde, } \\
\text { The Netherlands }\end{array}$ & $\begin{array}{l}\text { Waarde } \\
\text { (W2) }\end{array}$ & $\begin{array}{l}\text { Estuarine } \\
\text { fringe }\end{array}$ & 110 & $\begin{array}{l}\text { Linear } \\
\text { dendritic }\end{array}$ & Broad & $\begin{array}{l}\text { Puccinellia } \\
\text { maritima }\end{array}$ & Mud \\
\hline $\begin{array}{l}\text { Loire, } \\
\text { France }\end{array}$ & $\begin{array}{l}\text { Canal du Syl } \\
\text { (L3-L5) subtidal }\end{array}$ & Semi-natural & 200 & $\begin{array}{l}\text { Super- } \\
\text { imposed }\end{array}$ & Narrow & $\begin{array}{l}\text { Scirpus } \\
\text { maritimus }\end{array}$ & Mud \\
\hline Mira, Portugal & $\begin{array}{l}\text { (M1) subtidal } \\
\text { (M2) subtidal } \\
\text { (M3) subtidal } \\
\text { (M4) subtidal } \\
\text { (M5) subtidal } \\
\text { (M6) subtidal } \\
\text { (M7) subtidal } \\
\text { (M8) subtidal }\end{array}$ & $\begin{array}{l}\text { Estuarine } \\
\text { fringe }\end{array}$ & 10 & $\begin{array}{l}\text { Linear } \\
\text { dendritic }\end{array}$ & Narrow & $\begin{array}{l}\text { Spartina } \\
\text { maritima }\end{array}$ & Mud/Sand \\
\hline Cadiz, Spain & $\begin{array}{l}\text { Sancti Petri } \\
\text { (C1) }\end{array}$ & $\begin{array}{l}\text { Open } \\
\text { embayment }\end{array}$ & 625 & Complex & Broad & $\begin{array}{l}\text { Spartina } \\
\text { maritima }\end{array}$ & Mud \\
\hline
\end{tabular}

Rigaud 1984). Seasonal variation of the ichthyofauna and environmental parameters of the lower Loire weredescribed by Marchand (1980).

Mira estuary (Latitude: $\left.37^{\circ} \mathbf{4 0} \mathbf{N}\right)$ : The studied marsh is situated $4 \mathrm{~km}$ from the mouth of the estuary and is dominated by Spartina maritima and Anthrocnenom perenem. The marshes in the area develop over thin fringes of marginal sedimentary formations. All samples from this site were collected in the subtidal marsh ecotone, along the marsh edge, following a salinity gradient. The use of eelgrass beds and saltmarsh borders by fish in the Mira was described by Costa et al. (1994), although the data used in our study have not previously been published. A $1.7 \mathrm{~m}$ wide beam trawl was employed to sample a surface of approximately $1700 \mathrm{~m}^{2}$ per trawl.

Bay of Cadiz (Latitude: $36^{\circ} 30^{\prime} \mathbf{N}$ ): The data on the Cadiz marsh have been published by Drake \& Arias (1991a,b). The intertidal site (C1) was a tributary to the Sancti Petri Channel of the marsh. The other site (C2) is a subtidal marsh channel (Rio San Pedro) where water depth at low tide is about $1.3 \mathrm{~m}$ and where extensive tidal flats occur. Both tidal channels were sampled with $0.5 \mathrm{~mm}$ meshed stow nets. Both sites are polyhaline/euhaline and may become hyperhaline during warm summer months.

The study area was originally a part of the Guadalete river estuary but a sedimentary process and the construction of dams have changed the area into a marine marsh system, with insignificant freshwater inflow. The northeastern area of the marsh is crossed by the $12 \mathrm{~km}$ Rio San Pedro inlet, while the southeastern marsh is crossed by the sinuous $18 \mathrm{~km}$ long Sancti Petri Channel. A complex system of tidal channels and creeks supplies seawater to saltmarsh aquaculture systems situated along their courses. The gates of these lagoonal systems remain open, mainly in winter to early spring, to permit the tidal transport of fish larvae. During the rest of the year, free exchange of water with the adjacent tidal channels only takes place at spring high tides. The surface areas of 


\begin{tabular}{|c|c|c|c|c|c|c|c|c|}
\hline $\begin{array}{l}\text { Tidal } \\
\text { range } \\
(\mathrm{m})\end{array}$ & $\begin{array}{l}\text { Creek } \\
\text { width } \\
\text { (m) }\end{array}$ & $\begin{array}{l}\text { Creek } \\
\text { depth } \\
(\mathrm{m})\end{array}$ & $\begin{array}{l}\text { Salinity } \\
\text { range } \\
\text { (psu) }\end{array}$ & $\begin{array}{l}\text { Temp. } \\
\text { range } \\
\left({ }^{\circ} \mathrm{C}\right)\end{array}$ & $\begin{array}{c}\mathrm{O}_{2} \\
\text { (mg } \mathrm{l}^{-1} \text { unless } \\
\text { \% sat. given) }\end{array}$ & $\begin{array}{l}\text { Sample } \\
\text { period } \\
(\mathrm{mo} / \mathrm{yr})\end{array}$ & $\begin{array}{l}\text { Marsh } \\
\text { sampling } \\
\text { methods }\end{array}$ & $\begin{array}{l}\text { Reference site } \\
\text { sampling methods }+ \\
\text { mesh size (site codes) }\end{array}$ \\
\hline 4 & 3 & 1.5 & $11-32$ & $3-18$ & $\mathrm{n} / \mathrm{s}$ & 02/94-05/95 & $\begin{array}{l}\text { Block net } \\
8 \mathrm{~mm}\end{array}$ & $\begin{array}{l}\text { Beam trawl } \\
8 \mathrm{~mm}(\mathrm{~F} 2, \mathrm{~F} 3)\end{array}$ \\
\hline 7 & 1.75 & 0.5 & $25-32$ & $6-16$ & $85-118 \%$ & $07 / 96-08 / 96$ & $\begin{array}{l}\text { Fyke net } 15 \mathrm{~mm} \\
\text { Block net } 8 \mathrm{~mm}\end{array}$ & $\begin{array}{l}\text { Beam trawl } \\
8 \mathrm{~mm}(\mathrm{H} 2, \mathrm{H} 3)\end{array}$ \\
\hline 5 & 36 & 4 & $3-16$ & $6-23$ & $1.4-5.5$ & $03 / 90-10 / 91$ & Stow net $1 \mathrm{~mm}$ & $\begin{array}{l}\text { Beam trawl } \\
5 \mathrm{~mm}(\mathrm{~W} 3)\end{array}$ \\
\hline 5 & 20 & 2.5 & $15-23$ & $2-21$ & $6.5-10.0$ & 03/90-08/91 & Stow net $1 \mathrm{~mm}$ & \\
\hline 2.2 & 5 & 3 & $0-10$ & $10-25$ & $0.5-8.6$ & 03/83-12/83 & Fyke net $5-10 \mathrm{~mm}$ & $\begin{array}{l}\text { Eel tow net } \\
0.8 \mathrm{~mm}(\mathrm{~L} 1, \mathrm{~L} 2)\end{array}$ \\
\hline 0 & 2.3 & 2 & & & & & Fyke net $5-10 \mathrm{~mm}$ & $\begin{array}{l}\text { Lift net } 5 \mathrm{~mm} \\
(\mathrm{~L} 1, \mathrm{~L} 2)\end{array}$ \\
\hline 0 & 2.5 & 0.5 & & & & & Fyke net $5-10 \mathrm{~mm}$ & \\
\hline 4 & - & - & $7-34$ & $8-27$ & $\mathrm{n} / \mathrm{s}$ & 07/91-06/92 & Beam trawl $10 \mathrm{~mm}$ & No reference sites \\
\hline 4 & - & - & $10-35$ & $8-26$ & $\mathrm{n} / \mathrm{s}$ & & & \\
\hline 4 & - & - & $13-30$ & $9-26$ & $\mathrm{n} / \mathrm{s}$ & & & \\
\hline 4 & - & - & $15-30$ & $9-27$ & $\mathrm{n} / \mathrm{s}$ & & & \\
\hline 4 & - & - & $20-34$ & $10-26$ & $\mathrm{n} / \mathrm{s}$ & & & \\
\hline 4 & - & - & $20-35$ & $13-26$ & $\mathrm{n} / \mathrm{s}$ & & & \\
\hline 4 & - & - & $25-35$ & $12-23$ & $\mathrm{n} / \mathrm{s}$ & & & \\
\hline 4 & - & - & $28-35$ & $12-23$ & $\mathrm{n} / \mathrm{s}$ & & & \\
\hline 3.5 & 15 & 1.2 & $29-46$ & $10-28$ & $\mathrm{n} / \mathrm{s}$ & $01 / 84-12 / 88$ & Stow net $0.5 \mathrm{~mm}$ & No reference sites \\
\hline 3.5 & 200 & 4.5 & $34-41$ & $12-26$ & $\mathrm{n} / \mathrm{s}$ & $04 / 88-04 / 89$ & Stow net $0.5 \mathrm{~mm}$ & \\
\hline
\end{tabular}

the systems are about 330 and 1980 ha in the Rio San Pedro and Sancti Petri Channel marshes respectively. There is a zonation in the marsh vegetation: Spartina maritima dominates the lower marsh while Sarcocornia spp. (S. fruticosa and S. perennis) and Arthrocnemum macrostachyum are the dominant species higher up the marsh.

Data manipulation and analysis. Where possible, all taxa were determined to species level. Of the taxa which were not determined to species level in individual datasets, only 'Gobiidae indet.' was recorded in 2 estuaries, the Humber and the Westerschelde. As this taxon record formed a significant component of the assemblage in the Humber estuary but not in the Westerschelde, it was decided to include it in the analysis as a single taxon. Treatment of synonyms and taxonomy was adopted from Elliott \& Dewailly (1995).

A classification of species into a number of functional guilds was taken from Elliott \& Dewailly (1995). Each species was assigned to an ecological guild, a vertical distribution guild and a dietary preference guild. In addition, those species assigned to benthic or demersal vertical distribution guilds were also assigned to a substratum preference guild. These guilds, described fully by Elliott \& Dewailly (1995), are outlined below. The present study, however, also included records for an additional 33 taxa not included in that work. The majority of these taxa were assigned to functional guilds (Table 2) on the basis of biological and life-history information in Wheeler (1978). A few taxa were not described by Wheeler but are also found in South African tidal marshes and were assigned to guilds on the basis of a similar classification exercise underway there (A. Paterson \& A. Whitfield pers. comm.). Reproductive guilds, as defined by Elliott \& Dewailly (1995), were not regarded as a useful category in this study, since a high proportion of individuals in many sites belonged to species which are not likely to reproduce in the tidal marsh or even estuarine environments. Some guild classifications employed by Elliott \& De- 
Table 2. Functional guild classifications for taxa not classified by Elliott \& Dewailly (1995) (/: pelagic species, therefore substratum preference not classified; guild codes see 'Material and methods' section)

\begin{tabular}{|c|c|c|c|c|c|}
\hline Family & Scientific name & $\begin{array}{l}\text { Ecological } \\
\text { guild }\end{array}$ & $\begin{array}{l}\text { Vertical } \\
\text { distribution } \\
\text { guild }\end{array}$ & $\begin{array}{l}\text { Substratum } \\
\text { preference } \\
\text { guild }\end{array}$ & $\begin{array}{l}\text { Dietary } \\
\text { preference } \\
\text { guild }\end{array}$ \\
\hline Batrachoididae & Halobatrachus didactylus & MA & $\mathrm{B}$ & M & IF \\
\hline \multirow[t]{3}{*}{ Blenniidae } & Lipophrys pholis & MA & $\mathrm{B}$ & $\mathrm{M}$ & IS \\
\hline & Lipophrys trigloides & MA & $\mathrm{B}$ & M & IS \\
\hline & Parablennius spp. & MA & B & $\mathrm{R}$ & IS \\
\hline Carangidae & Caranx rhonchus & MJ & $\mathrm{D}$ & $\mathrm{F}$ & IF \\
\hline Carangidae & Caranx spp. & MJ & $\mathrm{D}$ & / & IF \\
\hline Centrarchidae & Lepomis gibbosus & FW & $\mathrm{D}$ & MV & IS \\
\hline Clupeidae & Clupeoid larvae & MJ & $\mathrm{P}$ & / & PS \\
\hline Cyprinidae & Cyprinidae indet. & FW & $\mathrm{D}$ & MV & IF \\
\hline Fundulidae & Fundulus heteroclitus & ER & $\mathrm{P}$ & / & IS \\
\hline Gobiesocidae & Diplecogaster bimaculata & MA & B & $\mathrm{M}$ & IS \\
\hline \multirow[t]{2}{*}{ Gobiidae } & Gobiidae indet. & ER & $\mathrm{B}$ & $\mathrm{S}$ & IS \\
\hline & Pomatoschistus marmoratus & MA & $\mathrm{B}$ & $\mathrm{S}$ & IS \\
\hline Hemiramphidae & Hyporhamphus picarti & MA & $\mathrm{P}$ & / & IS \\
\hline Ictaluridae & Ictalurus melas (= Ameiurus melas) & FW & $\mathrm{B}$ & $\mathrm{F}$ & $\mathrm{CS}$ \\
\hline Mugilidae & Mugilidae indet. & MS & $\mathrm{P}$ & / & $\mathrm{HC}$ \\
\hline Mullidae & Mullus barbatus & MA & B & $\mathrm{R}$ & $\mathrm{CS}$ \\
\hline Percichthyidae & Dicentrarchus punctatus & MA & $\mathrm{D}$ & $\mathrm{M}$ & IF \\
\hline Pomatomidae & Pomatomus saltator & MJ & $\mathrm{P}$ & / & FS \\
\hline Rajidae & Raja undulata & MA & B & $\mathrm{S}$ & ISS \\
\hline \multirow[t]{2}{*}{ Scophthalmidae } & Lepidorhombus boscii & MA & $\mathrm{B}$ & $\mathrm{F}$ & $\mathrm{CS}$ \\
\hline & Lepidorhombus whiffiagonis & MA & $\mathrm{B}$ & $\mathrm{F}$ & CS \\
\hline Serranidae & Serranus hepatus & MA & $\mathrm{D}$ & RV & IF \\
\hline \multirow[t]{2}{*}{ Soleidae } & Dicologoglossa hexophthalma & MA & $\mathrm{B}$ & $\mathrm{S}$ & IS \\
\hline & Monochirus hispidus & MA & $\mathrm{B}$ & $\mathrm{S}$ & IS \\
\hline \multirow[t]{6}{*}{ Sparidae } & Dentex dentex & MA & $\mathrm{D}$ & $\mathrm{R}$ & FS \\
\hline & Dentex macrophthalmus & MA & $\mathrm{D}$ & $\mathrm{R}$ & $\mathrm{CS}$ \\
\hline & Diplodus bellottii & MJ & $\mathrm{D}$ & SV & IS \\
\hline & Diplodus puntazzo & MJ & $\mathrm{D}$ & SV & IS \\
\hline & Pagrus pagrus & MJ & $\mathrm{D}$ & $\mathrm{M}$ & $\mathrm{CS}$ \\
\hline & Sarpa salpa & MJ & $\mathrm{D}$ & MV & $\mathrm{HC}$ \\
\hline Torpedinidae & Torpedo torpedo & MA & $\mathrm{B}$ & $\mathrm{F}$ & IS \\
\hline
\end{tabular}

wailly were modified for individual taxa in this study on the basis of an improved understanding of the ecology of species using tidal marshes. The ecological guild of Gasterosteus aculeatus, present year-round in most tidal marsh systems in this study, was changed from 'CA' to 'ER' and the dietary preferences of Clupea harengus, present in these estuaries largely as a planktivorous juvenile, from 'IF' to 'PS'.

Ecological guilds contained truly estuarine resident species (ER), marine adventitious visitors (MA), diadromous (catadromous/anadromous) migrants (CA), marine seasonal migrants (MS), marine juvenile migrants ('nursery' species) (MJ), or freshwater adventitious visitors (FW).

Vertical distribution guilds were differentiated according to pelagic species (P), demersal species (D) and benthic species (B).

Substratum preference guilds included species found solely on sandy bottom (S), on fine/soft sediments ( $F$, sand, mud and/or fine gravel) rough bottom ( $R$, rocks, stones, pebbles) and on mixed or various bottom types ( $M$, species living indiscriminately on any bottom type).
An additional 4 guilds were designated for those species with a preference for vegetation on any particular substratum type (SV, FV, RV, MV).

Dietary preference guilds were considered strictly planktivorous (PS), strictly invertebrate feeders (IS), strictly piscivorous (FS), feeding on invertebrates and fishes (IF), carnivorous (CS) other than PS, IS, FS or IF or herbivorous/carnivorous (HC) but not omnivorous (OV).

Data were available in either species abundance or species density per unit area or unit volume, depending on the sampling methods employed. Species densities or abundance for each individual site were summed for the entire time series to provide a single value for each species per site. The same analytical methodology employed by Elliott \& Dewailly (1995) was used to compare sites, although cluster analysis here was based on PRIMER software (Clarke \& Warwick 1994). Clusters were determined on the basis of Bray-Curtis similarities, with distances calculated by group-average sorting. For quantitative analysis, summed data were converted to percent composition 
for each species. Binary analyses were performed on data transformed to 'presence/absence' format, while quantitative analyses of percent composition matrices were performed on untransformed data.

\section{RESULTS}

\section{Taxonomic distribution}

Table 3 provides a summary of the distribution of 106 fish species (or other lowest identified taxa) and 44 family records from the 6 estuarine systems included in this study. A total of 97 species, in 40 families, were recorded in the 22 tidal marsh sites included in this study. Of these, 22 species (15 families) were recorded in at least 1 of the tidal marsh sites and 1 of the 10 reference sites. An additional 9 species, in 4 families, were recorded exclusively in the subtidal reference sites. No species were common to the 6 tidal marsh systems or the estuary sites. Only 2 species (Anguilla anguilla and Platichthys flesus) were recorded in 5 marsh systems. Another 6 species (Gasterosteus aculeatus, Aphia minuta, Pomatoschistus minutus, Dicentrarchus labrax, Solea solea and Syngnathus acus) were recorded in 4 estuaries. Of these, only 2 (G. aculeatus and D. labrax) were recorded in both marsh and estuary sites in all 4 estuaries. At the family level, only the Gobiidae was recorded in all estuarine systems and in 5 of the marsh systems, being absent from the Loire marsh sites. The widest latitudinal ranges were held by 2 species $(A$. anguilla and $S$. acus) recorded in the most northerly (Forth) and southerly (Bay of Cadiz) systems.

\section{Analysis of taxonomic similarity}

Assessment of the similarity between sites based on taxonomic classification was undertaken for both binary (presence/absence) and quantitative (percent composition) data. Analysis of species' presence/absence data showed a clear major division into 3 latitudinally separate groups of sites, with low similarity between groups (Fig. 2a: Bray-Curtis Similarity, BCS < $20 \%$ ). The first group consisted of all southern European sites, from the Bay of Cadiz and the Mira estuary. These 2 estuaries were clearly separated at a relatively low level of similarity within the group (BCS $<40 \%$ ). The fish assemblages of the 2 most marine-influenced sites in the Mira (M7 and M8) were relatively dissimilar from the other Mira sites $(\mathrm{BCS} \approx 50 \%$ ). All Loire sites were grouped at a minimum similarity of $\mathrm{BCS} \approx 40 \%$. The downstream Loire sites (L1 to L3), below and just above the lower impounding dam, grouped very tightly at $>90 \%$ similarity. The third group consisted of all northern European sites (Westerschelde, Humber and Forth), with 3 groups clearly separated within it. The intertidal Humber and Westerschelde marsh sites form a relatively dissimilar grouping (BCS $<40 \%$ ). The 3 Forth sites are relatively similar (BCS $\approx 70 \%$ ) and the subtidal reference sites from the Westerschelde and Humber form the last sub-group at about $50 \%$ similarity. o. Species - binary data

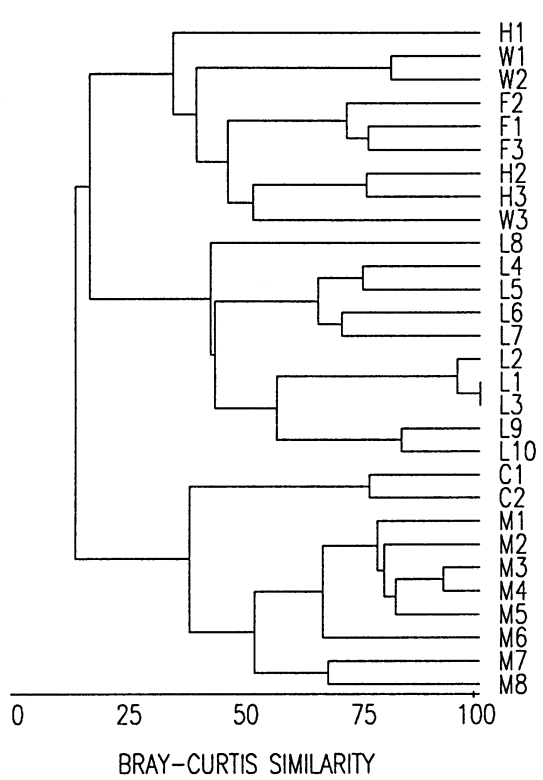

b. Species- percent composition

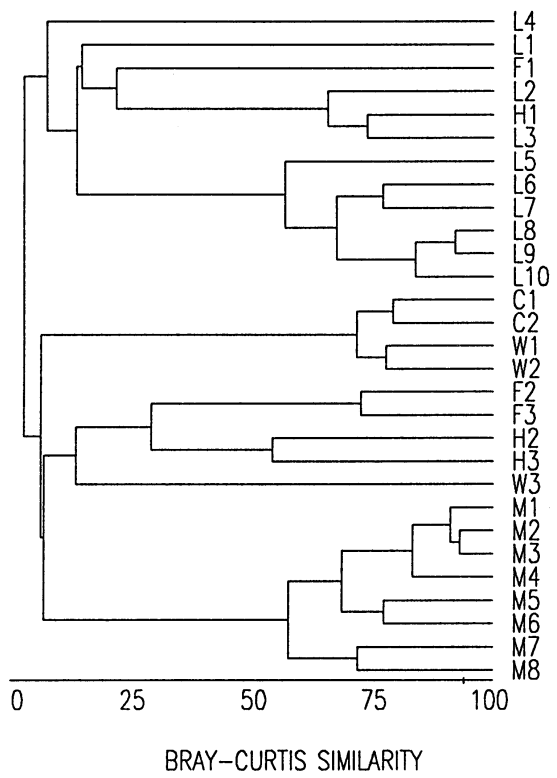

c. Families - binary dato

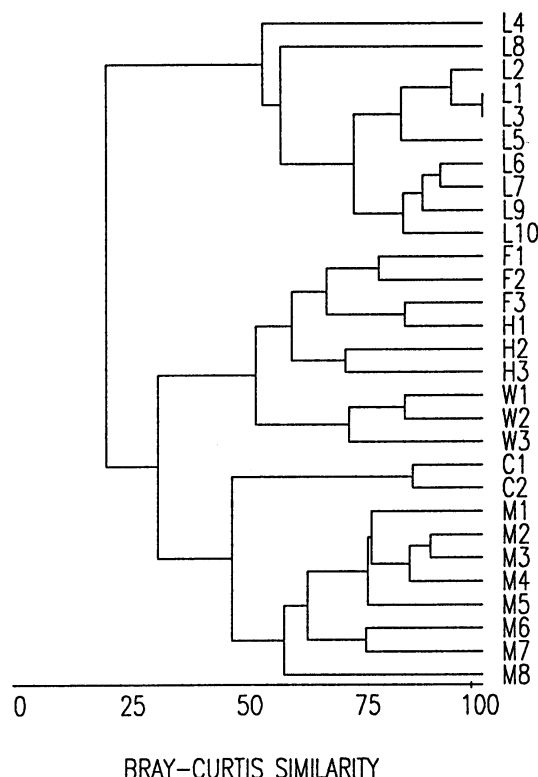

Fig. 2. Similarity analysis of taxonomic data: (a) species presence; (b) species percent composition; (c) family presence. Site codes see Table 1 
Table 3. Species records in estuary datasets (F, Forth; H, Humber; W, Westerschelde; L, Loire; M, Mira; C, Bay of Cadiz). (๑) Recorded only in tidal marsh site(s); $(\odot)$ Recorded in both marsh and estuary sites; (O) recorded only in estuary reference site(s)

\begin{tabular}{|c|c|c|c|c|c|c|c|}
\hline Family & Species/taxon & $\mathrm{F}$ & $\mathrm{H}$ & $\mathrm{W}$ & $\mathrm{L}$ & M & $\mathrm{C}$ \\
\hline Agonidae & Agonus cataphractus & 0 & 0 & 0 & - & - & - \\
\hline Ammodytidae & Ammodytes tobianus & - & - & & - & - & 0 \\
\hline Anguillidae & Anguilla anguilla & 0 & - & $\odot$ & $\odot$ & - & - \\
\hline \multirow[t]{2}{*}{ Atherinidae } & Atherina boyeri & - & - & - & - & - & 0 \\
\hline & Atherina presbyter & - & _- & - & - & ○ & - \\
\hline Batrachoididae & Halobatrachus didactyllus & - & - & - & - & ? & - \\
\hline Belonidae & Belone belone & - & - & - & - & - & - \\
\hline \multirow[t]{3}{*}{ Blenniidae } & Lipophrys pholis & - & - & - & - & - & 0 \\
\hline & Lipophrys trigloides & - & - & - & - & - & 0 \\
\hline & Parablennius spp. & - & - & - & - & - & - \\
\hline Callionymiidae & Callionymus lyra & - & - & - & - & 0 & - \\
\hline \multirow{3}{*}{ Carangidae } & Caranx rhonchus & - & - & - & - & - & - \\
\hline & Caranx spp. & - & - & - & - & - & - \\
\hline & Trachurus trachurus & - & - & - & - & $\bullet$ & - \\
\hline Centrarchidae & Lepomis gibbosus & - & - & - & $\odot$ & - & - \\
\hline \multirow{5}{*}{ Clupeidae } & Alosa fallax & - & - & 0 & - & - & - \\
\hline & Clupea harengus & $\odot$ & 0 & $\odot$ & - & - & - \\
\hline & Clupeoid larvae & - & 0 & • & - & - & - \\
\hline & Sardina pilchardus & - & - & - & - & - & 0 \\
\hline & Sprattus sprattus & $\odot$ & $\odot$ & $\odot$ & - & - & - \\
\hline Congridae & Conger conger & - & - & - & - & $\bullet$ & - \\
\hline \multirow{8}{*}{ Cyprinidae } & Abramis brama & - & - & - & - & - & - \\
\hline & Alburnus alburnus & - & - & $\bullet$ & - & - & - \\
\hline & Cyprinidae indet. & - & - & - & $\odot$ & - & - \\
\hline & Cyprinus carpio & - & - & - & 0 & - & - \\
\hline & Leuciscus leuciscus & - & - & - & 0 & - & - \\
\hline & Rutilus rutilus & - & - & - & 0 & - & - \\
\hline & Scardinius erythrophthalmus & - & - & - & 0 & - & - \\
\hline & Tinca tinca & - & - & - & 0 & - & - \\
\hline Cyprinodontidae & Fundulus heteroclitus & - & - & - & - & - & ? \\
\hline Engraulidae & Engraulis encrasicolus & - & - & - & - & - & - \\
\hline Esocidae & Esox lucius & - & - & - & $\bullet$ & - & - \\
\hline \multirow[t]{5}{*}{ Gadidae } & Ciliata mustela & - & - & 0 & - & 0 & - \\
\hline & Gadus morhua & $\bullet$ & 0 & 0 & - & - & - \\
\hline & Merlangius merlangus & $\odot$ & 0 & $\odot$ & - & - & - \\
\hline & Pollachius virens & - & $\bullet$ & - & - & - & - \\
\hline & Trisopterus luscus & - & 0 & 0 & - & - & - \\
\hline \multirow[t]{2}{*}{ Gasterosteidae } & Gasterosteus aculeatus & $\odot$ & $\odot$ & $\odot$ & 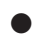 & - & - \\
\hline & Pungitius pungitius & - & - & $\bullet$ & $\odot$ & - & - \\
\hline Gobiesocidae & Diplecogaster bimaculata & - & - & - & - & - & - \\
\hline \multirow[t]{9}{*}{ Gobiidae } & Aphia minuta & - & 0 & - & 0 & 0 & 0 \\
\hline & Gobiidae indet. & - & 0 & $\odot$ & - & - & - \\
\hline & Gobius niger & - & - & - & - & - & - \\
\hline & Gobius paganellus & - & - & - & - & - & 0 \\
\hline & Pomatoschistus lozanoi & - & - & $\odot$ & - & - & - \\
\hline & Pomatoschistus marmoratus & - & - & - & - & $\bullet$ & - \\
\hline & Pomatoschistus microps & - & - & $\odot$ & 0 & 0 & - \\
\hline & Pomatoschistus minutus & $\odot$ & - & 0 & 0 & 0 & - \\
\hline & Pomatoschistus pictus & - & - & 0 & - & ? & - \\
\hline Hemiramphidae & Hyporhamphus picarti & - & - & - & - & - & 0 \\
\hline Ictaluridae & Ictalurus melas & - & - & - & ○ & - & - \\
\hline \multirow[t]{2}{*}{ Labridae } & Symphodus bailloni & - & - & - & - & $\bullet$ & 0 \\
\hline & Symphodus melops & - & - & - & - & 0 & - \\
\hline Liparidae & Liparis liparis & - & 0 & 0 & - & - & - \\
\hline \multirow[t]{6}{*}{ Mugilidae } & Chelon labrosus & - & - & - & - & - & - \\
\hline & Liza aurata & - & - & - & - & - & • \\
\hline & Liza ramada & - & - & - & $\odot$ & - & 0 \\
\hline & Liza saliens & - & - & - & - & - & 0 \\
\hline & Mugil cephalus & - & - & - & - & - & 0 \\
\hline & Mugilidae indet. & - & - & 0 & - & - & - \\
\hline \multirow{2}{*}{ Mullidae } & Mullus barbatus & - & - & - & - & - & - \\
\hline & Mullus surmuletus & - & - & - & - & 0 & 0 \\
\hline Osmeridae & Osmerus eperlanus & $\odot$ & $\odot$ & - & - & - & - \\
\hline
\end{tabular}


Table 3 (continued)

\begin{tabular}{|c|c|c|c|c|c|c|c|}
\hline Family & Species/taxon & $\mathrm{F}$ & $\mathrm{H}$ & $\mathrm{W}$ & $\mathrm{L}$ & $\mathrm{M}$ & $\mathrm{C}$ \\
\hline \multirow[t]{2}{*}{ Percichthyidae } & Dicentrarchus labrax & - & • & $\odot$ & - & - & $\bullet$ \\
\hline & Dicentrarchus punctatus & - & - & - & - & - & 0 \\
\hline Percidae & Stizostedion lucioperca & - & - & - & - & - & - \\
\hline Petromyzonidae & Lampetra fluviatilis & - & - & 0 & - & - & - \\
\hline \multirow[t]{3}{*}{ Pleuronectidae } & Limanda limanda & - & 0 & 0 & - & - & - \\
\hline & Platichthys flesus & $\odot$ & $\odot$ & $\odot$ & $\odot$ & - & - \\
\hline & Pleuronectes platessa & $\odot$ & $\mathrm{S}$ & $\odot$ & - & - & - \\
\hline Poecilidae & Gambusia affinis & - & - & - & $\odot$ & - & - \\
\hline Pomatomidae & Pomatomus saltator & - & - & - & - & - & 0 \\
\hline \multirow[t]{2}{*}{ Rajidae } & Raja clavata & - & 0 & - & - & - & - \\
\hline & Raja undulata & - & - & - & - & $\bullet$ & - \\
\hline Salmonidae & Salmo trutta & - & - & - & - & - & - \\
\hline \multirow[t]{3}{*}{ Scophthalmidae } & Lepidorhombus boscii & - & - & - & - & - & - \\
\hline & Lepidorhombus whiffiagonis & - & - & - & - & - & - \\
\hline & Scophthalmus rhombus & - & - & - & - & - & 0 \\
\hline Serranidae & Serranus hepatus & - & - & - & - & $\bullet$ & 0 \\
\hline \multirow[t]{5}{*}{ Soleidae } & Dicologoglossa cuneata & - & - & - & - & - & 0 \\
\hline & Dicologoglossa hexophthalma & - & - & - & - & $\bullet$ & - \\
\hline & Monochirus hispidus & - & - & - & - & - & 0 \\
\hline & Solea senegalensis & - & - & - & - & $\bullet$ & 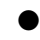 \\
\hline & Solea solea & - & 0 & $\odot$ & - & - & 0 \\
\hline \multirow[t]{11}{*}{ Sparidae } & Dentex dentex & - & - & - & - & $\bullet$ & - \\
\hline & Dentex macrophthalmus & - & - & - & - & - & 0 \\
\hline & Diplodus annularis & - & - & - & - & $\bullet$ & 0 \\
\hline & Diplodus bellottii & - & - & - & - & - & 0 \\
\hline & Diplodus puntazzo & - & - & - & - & - & 0 \\
\hline & Diplodus sargus & - & - & - & - & - & 0 \\
\hline & Diplodus vulgaris & - & - & - & - & $\bullet$ & 0 \\
\hline & Pagrus pagrus & - & - & - & - & $\bullet$ & 0 \\
\hline & Sarpa salpa & - & - & - & - & 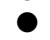 & 0 \\
\hline & Sparus aurata & - & - & - & - & 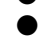 & 0 \\
\hline & Spondyliosoma cantharus & - & - & - & - & - & - \\
\hline \multirow[t]{7}{*}{ Syngnathidae } & Hippocampus hippocampus & - & - & - & - & $\bullet$ & 0 \\
\hline & Hippocampus ramulosus & - & - & - & - & - & 0 \\
\hline & Nerophis ophidion & - & - & - & - & - & 0 \\
\hline & Syngnathus abaster & - & - & - & - & - & 0 \\
\hline & Syngnathus acus & $\odot$ & - & 0 & - & $\bullet$ & 0 \\
\hline & Syngnathus rostellatus & - & - & $\odot$ & - & - & - \\
\hline & Syngnathus typhle & - & - & - & - & - & 0 \\
\hline Torpedinidae & Torpedo torpedo & - & - & - & - & - & - \\
\hline Trachinidae & Echiichthys vipera & - & 0 & - & - & - & - \\
\hline Triglidae & Trigla lucerna & - & - & 0 & - & - & - \\
\hline Zoarcidae & Zoarces viviparus & $\odot$ & - & 0 & - & - & - \\
\hline
\end{tabular}

Comparison of sites on the basis of percent composition at species level identifies 4 major, highly dissimilar groups (Fig. 2b). All 8 Mira sites form a relatively tight cluster at a similarity of approximately $50 \%$. Again, the 2 most saline sites (M7 and M8) separate clearly from the remaining sites. The second group is formed of the subtidal north European sites, which separate within this cluster into individual estuaries. The third group is a highly similar cluster of the Bay of Cadiz and Westerschelde marsh sites, which share a high dominance (>65\% composition) by a single species (Pomatoschistus microps). The fourth major group consists of the Forth and Humber intertidal marsh sites and all Loire sites. The upstream Loire sites (L5 to L10), connected at a similarity of about $50 \%$, are quite dissimilar to the other members $(\mathrm{BCS} \approx 15 \%)$ which are, themselves, relatively different. A single site from the Loire (L4), highly dissimilar to all other sites, was dominated (64\%) by Abramis brama and was the only Loire site where a fyke-type net with a $10 \mathrm{~mm}$ mesh was employed for sampling, in addition to the $5 \mathrm{~mm}$ mesh fyke net employed in most other Loire sites upstream of the impoundment (L4 to L10).

Cluster analysis of family presence/absence data (Fig. 2c) gives 3 groups of sites as separated, apparently on the basis of latitude. In this case, however, the northern European sites are more similar to the southern European sites $(\mathrm{BCS} \approx 25 \%$ ) than they are to the Loire sites $(\mathrm{BCS}<20 \%)$. Again, as with the species binary data, the southern grouping divides between 
the Mira and the Bay of Cadiz. The Westerschelde is separated at about $50 \%$ similarity from the British estuaries, with a division between intertidal and subtidal sites in the Humber. The Loire site (L4) is again quite dissimilar to all other sites in the estuary and the 3 sites downstream of the Loire impoundment (L1 to L3) are highly similar (BCS $\approx 90 \%$ ).

\section{Analysis of functional guilds}

Ecological guilds

The relative proportions of each guild varied between marsh sites within estuaries, between marsh sites and subtidal reference sites, and between estuaries (Fig. 3a). Cluster analysis of the percent composi-
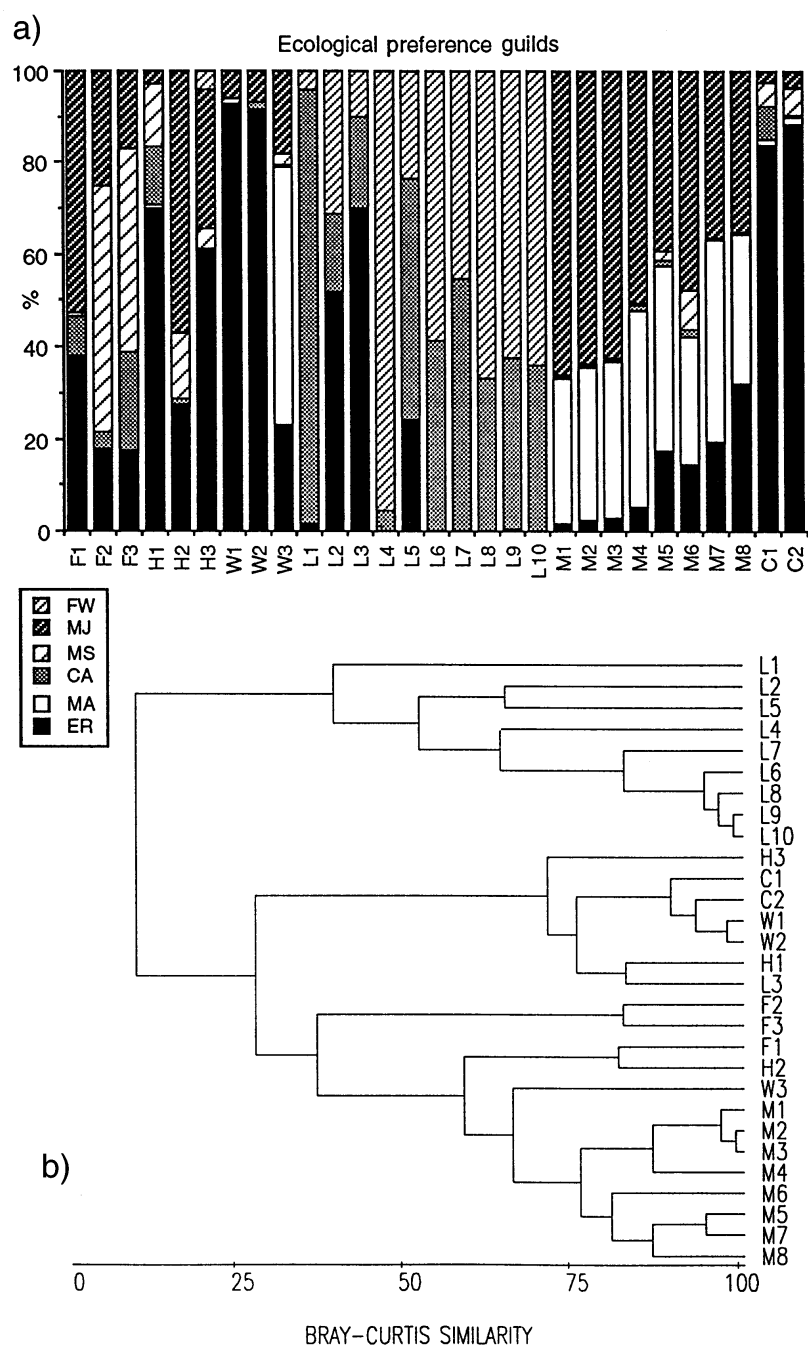

Fig. 3. Ecological guilds: (a) percent composition of each site assemblage; (b) Bray-Curtis similarity dendrogram. Guild codes see 'Material and methods' section tion of ecological guilds (Fig. 3b) indicated a major division ( $\mathrm{BCS} \approx 10 \%$ ) between almost all Loire sites and sites in other estuaries. The Loire, largely freshwater or very low salinity, was the only tidal marsh in the dataset (Fig. 3a) to have a high percentage of freshwater species (FW) and, in upstream sites (L6 to 10), a marked absence of estuarine resident (ER) and marine adventitious species (MA). Most Loire sites also had a relatively high percentage of diadromous species (CA). In the case of the Loire subtidal site L1, the dominance of this ecological category was as high as 95\% due to the extremely high abundance of the eel Anguilla anguilla. Assemblages of the sites in the remaining estuaries were largely composed of estuarine resident (ER) and marine species (MA, MS and $\mathrm{MJ})$.

Cluster analysis divided these latter sites at a low level of similarity (BCS $\approx 30 \%$, Fig. 3b). All Mira and Forth sites, and subtidal sites from the Humber and the Westerschelde, were separated from the Bay of Cadiz, the Westerschelde, marsh sites, the Humber marsh site, the second Humber subtidal site and the Loire site L3. The former group contains the sites which were largely dominated by marine juveniles and/or marine seasonal residents. The sites in the latter group were heavily dominated by estuarine residents with only a minor representation of other guilds.

\section{Vertical distribution guilds}

The re-grouping of species' percentage composition into 3 vertical distribution guilds (Fig. 4a: pelagic, demersal and benthic) revealed a major division (Fig. $4 \mathrm{~b}: \mathrm{BCS} \approx 40 \%$ ) between individual sites heavily dominated by benthic fish and sites dominated by both pelagic and benthic species. The Westerschelde, the Bay of Cadiz, the Humber, the Mira and 2 Loire sites belonged to the former category, while all sites in the Forth and the majority in the Loire were in the latter. The division was also apparent within the Loire, with sites furthest from the main estuary (L6 to L10) generally having a higher percentage of pelagic species. In this guild analysis, the Loire Site L4 is highly dissimilar to all other sites (Fig. $4 \mathrm{~b}: \mathrm{BCS}=20 \%$ ).

\section{Substratum preference guilds}

The division between substratum preference guilds is shown in Fig. 5a; those species with an additional preference for vegetated habitats were pooled with those of the main substratum type. For those species regarded as having a benthic or demersal distribution, the substratum preferences, as determined by 8 guilds, 

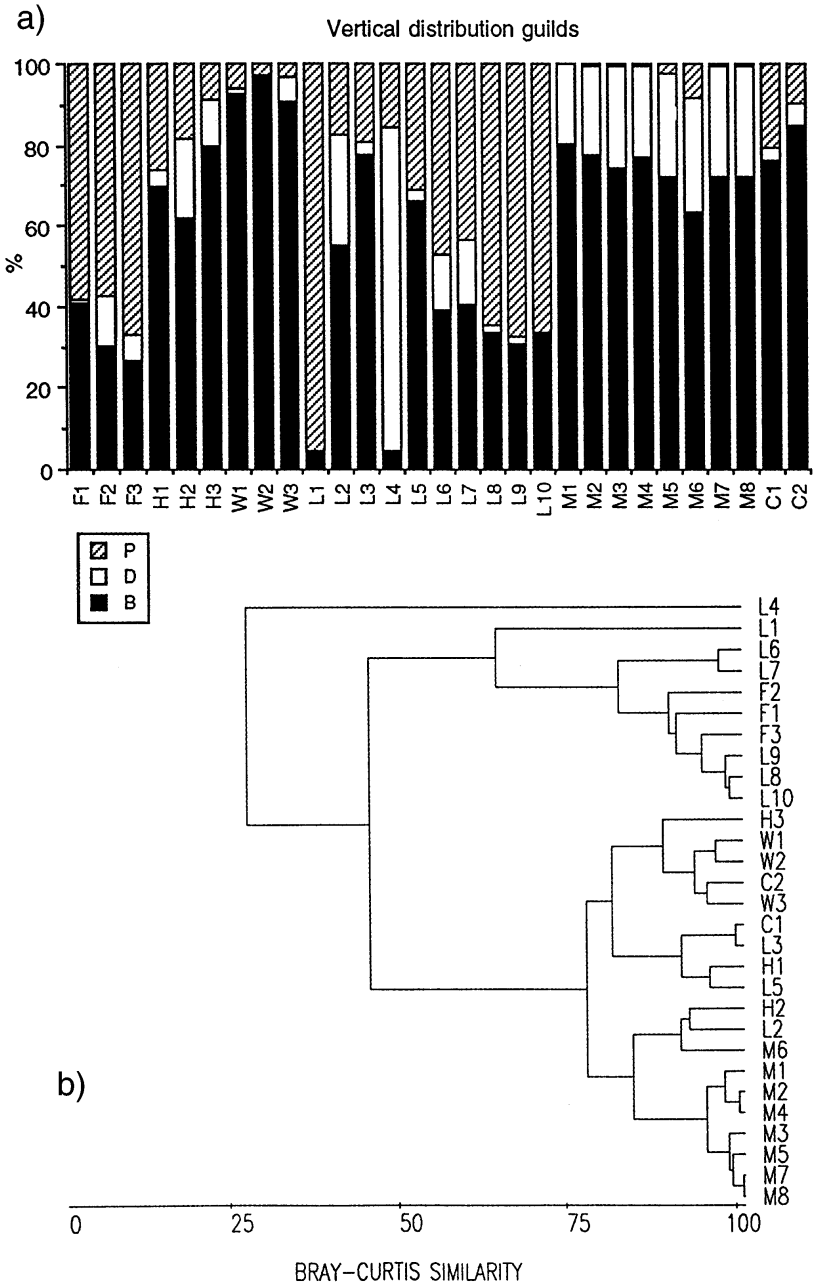

Fig. 4. Vertical distribution guilds: (a) percent composition of each site assemblage; (b) Bray-Curtis similarity dendrogram. Guild codes see 'Material and methods' section

are highly variable between estuaries, although usually consistent within estuaries (Fig. 5a).

Fish species preferring a hard substratum were largely absent in the investigated sites. A number of sites (Forth subtidal, Humber, Loire) were largely dominated by species whose substratum preference is indicated as 'fine sediments' (F). Others (Westerschelde, Bay of Cadiz, Forth intertidal) showed a strong dominance by species preferring 'sand' (S). The Mira estuary marsh sites had assemblages with a major preference for mixed substrata (M), in combination with species with a preference for fine sediments. Only upstream sites in the Loire (L5 to L10) had a large percentage of species with a preference for vegetated habitats in combination with a preference for fine sediments $(\mathrm{FV})$. These preferences are reflected in the cluster analysis of the guilds, with 3 major groupings consisting of the sites where the assemblages have rough/fine substratum preferences (Mira estuary), fine-dominated preferences (majority of Loire, Forth and Humber sites) and sand-dominated preferences (Bay of Cadiz and Westerschelde). Two Loire sites (L1 and L4) are highly different from all other sites. L1 had the lowest percentage of non-pelagic fish ( $4.5 \%$ of total) in any site.

\section{Dietary preference guilds}

The relative proportions of the dietary preference guilds generally varied greatly between estuaries while remaining relatively consistent within estuaries (Fig. 6a). Only 1 estuary, the Forth, had assemblages dominated by the planktivorous guild (PS) and a low percentage of strict invertebrate feeders (IS). Only a few other sites had a low complement of IS species (Humber marsh site, Loire Sites L1 to L5). The majority
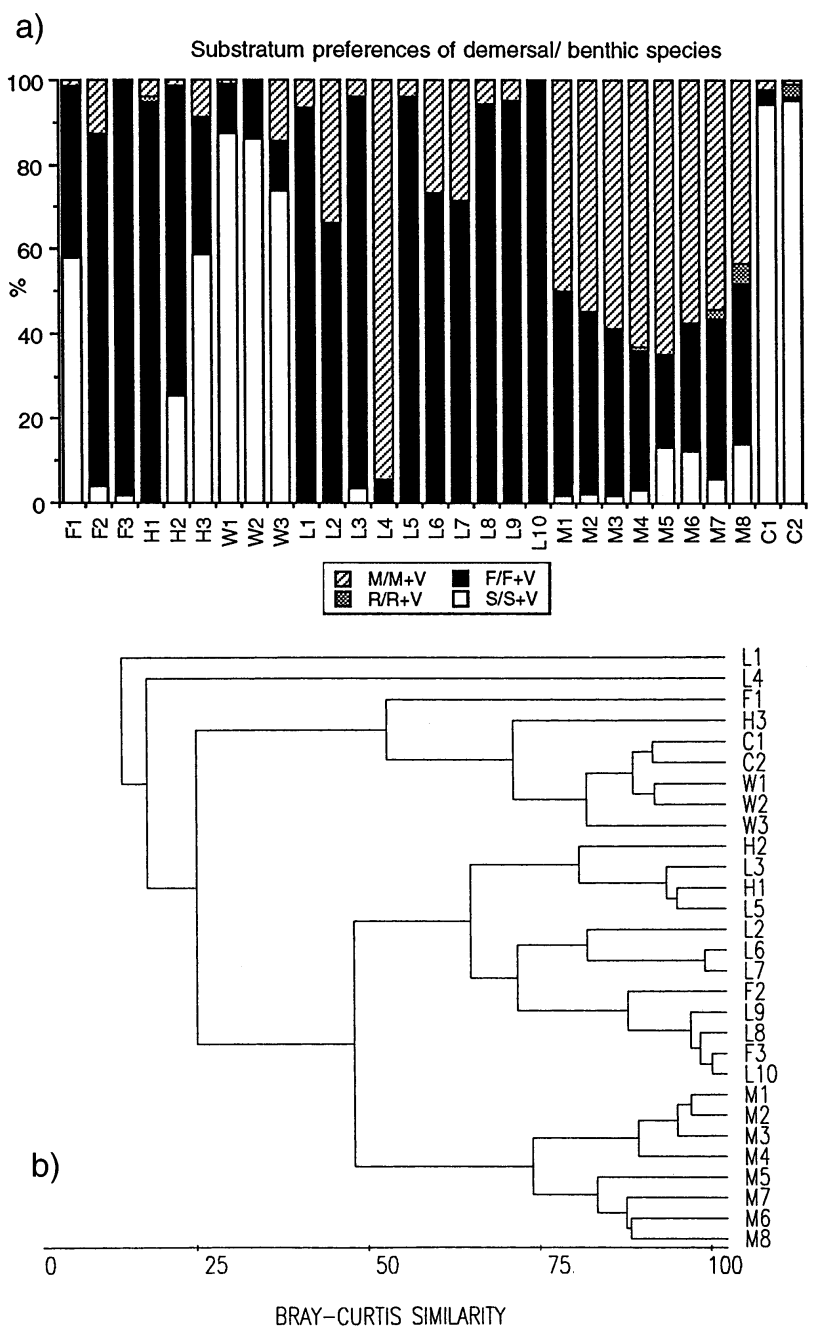

Fig. 5. Substratum preference guilds (for demersal and benthic taxa): (a) percent composition of each site assemblage; (b) Bray-Curtis similarity dendrogram. Guild codes see 'Material and methods' section 


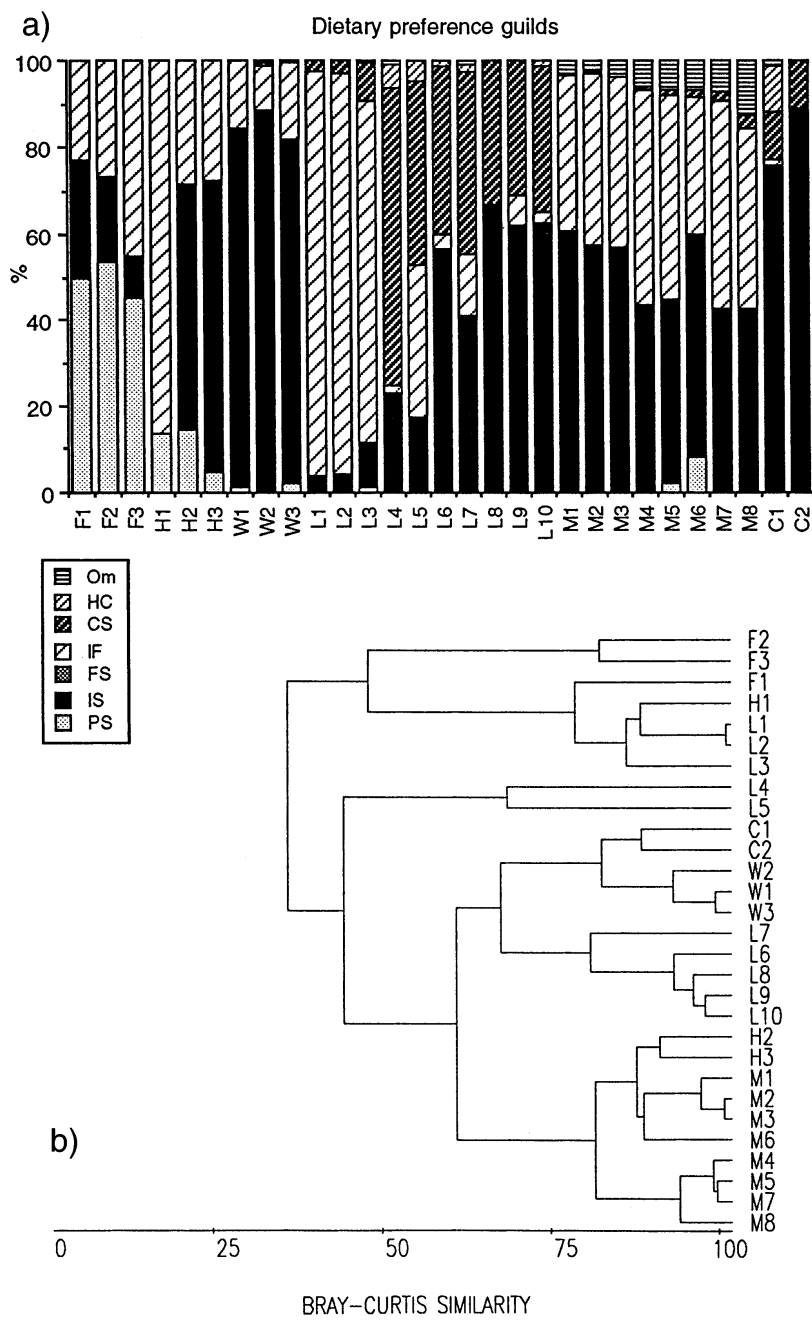

Fig. 6. Dietary preference guilds: (a) percent composition of each site assemblage; (b) Bray-Curtis similarity dendrogram. Guild codes see 'Material and methods' section

of other sites had a high percentage of IS feeders, with some also displaying high levels of general carnivorous feeders (CS) or invertebrate/fish feeders (IF). Only in the Mira estuary was there evidence of an omnivorous guild, present at low levels but increasing gradually from M1 to M8, towards the marine end of the estuary.

\section{DISCUSSION}

\section{General discussion}

This is believed to be the first attempt to produce a comprehensive, functional-guild-based assessment of tidal marsh fish assemblages for a large geographical region, incorporating aspects of salinity, habitat and dietary preferences and seasonality of use. Despite the variation in creek size between sampling sites, all the intertidal marsh sites included in this study are, by virtue of their location, relatively shallow. Also, while a few of the individual sites (Loire sites upstream of the impoundment) are not subject to regular twice-daily tidal inundation, the majority are truly tidal, with relatively unhindered access for fish in the subtidal areas of the estuary during the high water period. This means that the comparison undertaken here between the fish assemblages of the different marsh systems represents the comparison of the use by fish of similar physiographic features in each marsh.

In combining the data here, considerable constraints were imposed on data comparability. These data were collected over varying time periods by a number of sampling methods, with sample collection based on different sampling strategies and effort (Table 1), all of which constitute potential sources of variability between datasets. For example, it is difficult to determine to what degree the high incidence of Anguilla anguilla in some Loire sites may reflect the rather specialised sampling apparatus employed, developed originally for the capture of this species on a commercial basis. In their comparison of the fish assemblages of 17 European estuaries, Elliott \& Dewailly (1995) also acknowledged the problem of comparing data generated by a number of different sampling gears.

While facing similar problems with data comparability, the present study makes greater use of the information content of individual datasets by summing individual taxa abundance or density data for the entire study period at each site. As with Elliott \& Dewailly (1995), temporal aspects of the data are ignored. This approach has allowed the abundance of dominant taxa at each site to be taken into account in a way that was not possible for Elliott \& Dewailly (1995). In the present study, loss of information on spatial variability is not an issue. Compared to Elliott \& Dewailly's 'whole-estuary' approach, this study includes data from a limited number of habitats within each estuary.

A comparison of sites based on absolute abundance and density values was not likely to provide meaningful differences between sites. Where data on absolute abundance or density from different tidal marsh sites are comparable, such assessments have proven valuable elsewhere, generating hypotheses which sought to explain order-of-magnitude regional differences in nekton densities in salt marsh habitats (southeast USA: Zimmerman et al. 1991). In our study, the conversion of absolute values to percent composition has reduced the variability in absolute values resulting from methodological differences while still retaining the relative abundance of individual taxa within each individual site's dataset. In this way, it has been possible to 
compare the structure and functioning in terms of the relative importance of taxa or functional guilds at each site. To extend such an analysis to the comparison of differences in absolute densities (or biomass), as has been attempted in the USA (Zimmerman et al. 1991), will require greater co-ordination between researchers. The collection of further data on the use of European tidal marsh habitats by fish and other nekton would benefit from the adoption of a standardised set of methodologies, such as, for example, the mesh size(s) which should be used for nekton sampling.

Elliott \& Dewailly (1995) identified similar separations to those identified here, between the assemblages of Iberian estuaries and those of Dutch and UK North Sea estuaries. It was further suggested that this separation of sites may represent a division between Lusitanian and Boreal faunas. In that study, however, the Loire assemblage was identified as highly similar to those of the Iberian estuaries. Elliott \& Dewailly's study included data on fish assemblages from higher salinity areas in the Loire estuary, as compared to the low salinity marsh area in this study. In the present study, it is most likely the low-salinity nature of the Loire sites that leads to the lack of similarity of its marsh fish assemblages to those of either the southern or northern estuaries.

The general latitudinal trend in taxonomic composition in European estuarine fish assemblages demonstrated by Elliott \& Dewailly (1995), and here for tidal marsh assemblages, is also reflected by the scarcity of ubiquitous species in the tidal marshes. The 2 most widely recorded species, the flounder Platichthys flesus and the European eel Anguilla anguilla, are not confined to tidal marsh habitats but are to be found in most northern, western and European Mediterranean estuarine and lower river systems (Wheeler 1978, Maitland \& Campbell 1992). The presence of these species in the majority of the tidal marshes in this study does not necessarily, therefore, make them particularly distinctive indicators of tidal marsh assemblages. The absence of $A$. anguilla from the Humber marsh assemblage may simply be related to the lower sampling effort employed compared to the other studies combined here, as the species has been recorded in the Humber estuary (Marshall \& Elliott 1996, 1998). The absence of $P$. flesus from the Bay of Cadiz, within its recorded geographical range (Wheeler 1978), does seem to reflect a genuine absence from, or rarity in, the estuary (Drake pers. obs.).

The finding here that there is a level of similarity between tidal marsh assemblages of different estuaries at the family level, and at a higher level of similarity than for species in most cases, reinforces Costa \& Elliott (1991), who found such similarities between European estuaries, even where there was only limited similarity at the species level. Kneib (1997) indicates that 1 family of fishes, the Cyprinodontidae or killifishes, is among the dominant fishes in every intertidal marsh habitat under all salinity conditions, with the notable exceptions of Australia and New Guinea. The tidal marshes of Europe should be added to these exceptions, as only a single cyprinodont species, the mummichog Fundulus heteroclitus, is recorded as a very rare component of the intertidal assemblage from the Bay of Cadiz, where it is present as an introduced alien species. Its presence in the intertidal creek but not the subtidal is consistent with its known behaviour in North American salt marshes, where it tends to remain in intertidal areas at low tide, often using creeks as a residence (Bigelow \& Schroeder 1953, Halpin 1997). Another alien species is the poecilid species Gambusia affinis, recorded widely throughout the sites of the Loire estuary tidal marsh. This species was introduced to France in an attempt to control mosquito larvae at some point between 1927 (Allardi \& Keith 1991) and the late 1940s (Lever 1997), and was first recorded in the Loire around 1960 (Marchand pers. obs.). This species is numerically dominant in several wholly freshwater Loire marsh sites. In these sites, the fish assemblage must be regarded as strongly altered from that expected of a natural tidal marsh, both by the effects of enclosure and the proliferation of the alien $G$. affinis.

The ubiquity of members of the family Gobiidae in the European tidal marsh sites is consistent with the importance of this family in estuarine nekton assemblages generally (e.g. Allen \& Barker 1990, Potter et al. 1990) and, more specifically, in tidal marsh assemblages outside Europe (e.g. USA: Hackney \& de la Cruz 1981, Peterson \& Turner 1994; Australia: Davis 1988). Kneib (1997) reported that, as gobiid fishes resident in tidal marsh systems typically have relatively sedentary lifestyles, they are not effectively captured by most quantitative sampling methods. In consequence, relatively little is known of the habits of gobies in tidal marshes. Inefficient capture of gobies certainly occurred during sampling of the Humber intertidal marsh site in this study, where gobies Pomatoschistus microps were known to be present in the creek, as they were captured by bottle-trapping, but were absent from the fyke- and block-net samples and consequently were not included in this study (Gardner pers. obs.).

Given the relatively large mesh sizes (generally 8 to $15 \mathrm{~mm}$ ) of sampling nets employed in most of the tidal marsh studies included here (Forth, Humber, Loire, Mira), it is probable that smaller individual gobies were not sampled representatively in any of these sites. Indeed, the relatively small mesh sizes (0.5 to $1 \mathrm{~mm}$ ) employed in the Westerschelde and Bay of Cadiz marshes may account for the heavy dominance of the 4 tidal marsh assemblages in these 2 sites by a 
single goby species, Pomatoschistus microps. Comparison of species assemblages using percent composition data provides a less distinct separation of the 6 estuaries than that found using only binary data. The high degree of dominance (65 to $85 \%$ ) of the tidal marsh sites from the Westerschelde and the Bay of Cadiz by $P$. microps caused these 4 sites to group closely in both the species percent composition analysis and some of the guild-based comparisons. The tidal marsh fish assemblages of these 2 estuaries are, however, relatively dissimilar in terms of taxonomic composition, as shown by their separation when comparisons are made using 'species presence' data.

As described previously, the 'functional guilds' concept has been applied to both riverine and estuarine fish assemblages. Functional guilds have also been proposed to describe aspects of marine open-coast fish assemblages (Gibson \& Ezzi 1987, Wootton 1990). Previous approaches have typically been employed, either to provide values for metrics or to describe aspects of habitat-use by fish. Various authors have also applied certain of the guild categories to tidal marsh assemblages. In particular, the 'ecological guilds' were applied by Davis (1988) to the fish assemblages of a tidal swamp in northern Australia, where no marine adventitious and few freshwater adventitious species were noted to be using the habitat.

A general comparison between the results of Elliott \& Dewailly (1995) and the present study shows a much greater similarity between whole-estuary assemblages for each set of guilds than was found for tidal marsh assemblages. This is despite the considerably longer species' lists in individual estuary sites than in the 6 individual estuaries' tidal marsh assemblages. It should be noted that the methodology of Elliott \& Dewailly did not permit the relative abundance of different taxa to influence the between-estuary similarity scores. Their comparison was based on the total percentage of a site's species list within each guild category, rather than the percentage of the summed abundance for a site, as used in the present study. This difference would tend to exaggerate the differences between sites to a greater extent in the present study than in Elliott \& Dewailly's approach.

The ecological guilds originally proposed for estuarine fish by McHugh (1967) and subsequently developed by others (Haedrich 1983, Elliott \& Taylor 1989) represent a combination of salinity preferences or tolerances and behavioural traits, particularly in relation to migratory behaviour and seasonal use of the estuarine environment. Elliott \& Dewailly (1995) reported a typical European (Atlantic seaboard) estuarine fish assemblage as consisting of a majority equally of estuarine resident, marine adventitious and marine juveniles (25\% each), with a small number of marine seasonal migrant, diadromous and freshwater adventitious species. This 'typical assemblage' was constructed from samples of fish assemblages which excluded tidal marshes and from sites typically distributed along the salinity gradients of the estuaries. It would be surprising if tidal marsh assemblages generally reflected this proposed European 'whole-estuary' assemblage. Tidal marshes are frequently sited at restricted locations within estuaries, and at positions on the salinity gradient varying between estuaries, from polyhaline (e.g. Bay of Cadiz) to mesohaline (e.g. Forth, Mira, Humber) and even into oligohaline (e.g. Westerschelde) or freshwater (e.g. Loire) sections of estuaries.

The high dominance of both Westerschelde and Bay of Cadiz marsh sites by the estuarine resident goby species Pomatoschistus microps was not reflected in most other sites, where proximity to marine influence, signalled by the incursion of marine juvenile or marine seasonal species, was a more defining feature of the assemblages. The relative unimportance of the diadromous guild in almost all sites (except some Loire stations) may reflect the shallow-water nature of intertidal marshes or the relatively short period many diadromous species spend in estuarine areas during diadromy (McDowall 1988).

Tidal marsh fish assemblages might reasonably be expected to bear some similarity to that of nearby subtidal areas, particularly for intertidal areas of marshes where most fish are only present during high water periods, retreating subtidally with the ebb tide (Cattrijsse et al. 1994). This is particularly likely where tidal flow into marsh systems is unrestricted or only partially reduced by anthropogenic interference (Kneib 1997). The inclusion of limited data on nearby subtidal fish assemblages for 4 of the systems (all except Mira and Bay of Cadiz) allowed a comparison to be made between assemblages in marsh creeks and those in the larger bodies of estuarine water to which they are connected. In most cases, this connectivity was reflected by generally greater 'within-estuary' than 'betweenestuary' similarity values in the composition of ecological guilds.

As reported by Rozas (1993) for tidal marshes in the southeastern USA and reviewed more widely for tidal marshes world-wide by Kneib (1997), several individual sites in this study contained a large percentage of marine juvenile fish. This was particularly so for the Forth and the Mira, while the Humber intertidal marsh site had a considerably lower percentage of this guild than its 2 nearby subtidal reference sites. The importance of European marsh sites as a habitat for juvenile fish is further emphasised by the high incidence of estuarine residents in many sites. A large percentage of a guild that spends its entire life cycle in the estuary inevitably consists of juvenile individuals, as 
supported by the datasets in this study (Drake \& Arias 1991a,b, Cattrijsse et al. 1994, Costa et al. 1994, authors' unpubl. data).

The analyses based on vertical distribution guilds and substratum preference guilds (for demersal and benthic species) generally followed the approach of Elliott \& Dewailly (1995), with the exception of the addition of 4 vegetation preference categories for the substratum preference guilds. Concerning the vertical distribution guilds, the overwhelming majority of individuals in most sites were benthic dwellers, largely reflecting the lifestyles of the gobies and flatfish which made up much of the assemblage. Only the Forth estuary (both intertidal marsh and subtidal reference sites) and several of the Loire sites showed a dominance by pelagic species. The Forth estuary is known to be an important habitat for juvenile clupeoids Clupea harengus and Sprattus sprattus (Elliott \& Taylor 1989). The estimates of population sizes and production for these species have previously been reported as likely underestimates for the Forth as fish population sampling in the subtidal areas is undertaken by demersal trawling (Elliott \& Taylor 1989). The relative similarity of percent composition of pelagic species in all 3 sites in the Forth is surprising, therefore, as a block net in a tidal marsh creek is likely to sample this guild more effectively than a demersal trawl in the estuary channel.

The relatively complex structure of tidal marsh habitats compared to most subtidal habitats does offer the potential for influencing vertical zonation in the fish assemblage. The greatest water depth at any site, however, was only $4.5 \mathrm{~m}$, with intertidal sites generally less than $2 \mathrm{~m}$ deep at high water, which may be insufficiently deep for vertical structural influences to be significant. The value of the vertical distribution guild category for tidal marshes is not clear from this study, and it may require application to areas with much greater vertical ranges (i.e. a whole estuary) to provide informative classifications of fish assemblages. The classification of vertical distribution into 3 simple classes, benthic, demersal and pelagic, may be too simplistic for the structurally rich but shallow habitats of tidal marshes (see below).

Similarly, the value of the substratum preference guilds for exploring the use of tidal marshes by fish is not demonstrated clearly by this study. Using the Elliott \& Dewailly (1995) guild classification for Pomatoschistus microps as a species with a preference for sand habitats clearly provides a different analysis of the similarity of the Cadiz and Westerschelde sites than would be obtained if the substratum preferences observed in some marshes were used. In the Westerschelde, for example, P. microps is found extensively on fine sediments in environments where sand habitats are rare, if not absent (Cattrijsse pers. obs.). The same argument could be made for the sand goby $P$. minutus, or for juvenile plaice Pleuronectes platessa, both classed as preferring sandy substrata but abundant widely in the mesohaline, fine sediment environments of the middle stretches of the Forth estuary and its tidal marshes (Elliott \& Taylor 1989, Elliott et al. 1990, Mathieson pers. obs.). All tidal marshes in the current study are sited in areas of fine sediment and, consequently, the substratum preference guilds offer little prospect of explaining the role of sediment preferences in structuring the marsh assemblage. The only exception might be the inclusion of guilds describing additional preferences for vegetation on any substratum type. In this study, few species were accorded a preference for vegetated habitats other than certain the demersal freshwater species in the Loire and several species recorded in the Mira estuary and Bay of Cadiz. The value of substratum preference guilds is undoubtedly higher when considering the fish assemblage of a much larger area than was considered here, such as at the whole estuary level, or perhaps in habitats where a greater degree of substratum heterogeneity exists.

Dietary preference guilds indicated that the assemblages of most tidal marshes were dominated by species feeding on invertebrates (IS) or invertebrates and fish (IF). These guilds provide a relatively coarse definition of dietary preferences, particularly in relation to invertebrates, but are sufficient to distinguish between the majority of sites and the 3 sites from the Forth estuary, where the relative abundance of planktivorous clupeoid juveniles reduces the importance of the other guilds. Morton et al. (1987) employed a similar categorisation to describe the dietary preferences of fish in an Australian intertidal saltmarsh inlet as largely benthic feeders and only 1 major planktivorous feeder. In defining a typical European estuarine fish assemblage in dietary preference terms, Elliott \& Dewailly (1995) described, as here, an assemblage dominated by IF/IS guilds with relatively low levels of other carnivorous (CS/FS) and of herbivorous guilds. In both the estuarine and tidal marsh assemblages, the dietary preferences of the assemblages support the predominance of estuarine nekton food webs based on detritus, as proposed by da Sylva (1975). The percentages of carnivorous guilds (FS, CS) in the tidal marsh assemblages are generally lower than those observed for whole-estuary assemblages by Elliott \& Dewailly (1995), suggesting a potentially lower predation pressure on smaller fish inhabiting the tidal marshes, compared to subtidal estuarine areas outside the marsh. This pattern was reported in North Carolina by Rozas \& Hackney (1984), who collected only a few large predatory fish in intertidal creeks of an oligohaline marsh, although these were abundant in nearby subtidal areas. 
Shenker \& Dean (1979) similarly reported that they seldom collected large predatory fish species in a South Carolina salt marsh creek, although larval and juvenile fish were abundant.

\section{Further development of functional guilds for use in estuaries and tidal marshes}

Certain functional guilds have a clear and demonstrated value for describing the use of tidal marshes by fish and comparing the marsh assemblages with those of the main estuary. In particular, the ecological guilds proved useful for indicating the predominance of use by estuarine resident and marine juvenile species, and highlighted a strong effect of salinity on the tidal marsh assemblage. Kneib (1997) highlighted the potential importance to estuarine resident nekton of structure-rich tidal marsh habitats and reviewed the considerable literature describing the provision of a temporary habitat for marine juvenile fishes in tidal marshes. Kneib (1997) and others (e.g. Shenker \& Dean 1979) have also speculated upon the role of these habitats in providing a refuge from predation for these estuarine resident and marine juvenile fish. Despite some shortcomings of the dietary preference guilds, as described below, comparison of these guilds in European tidal marshes with those of overall estuarine fish assemblages (Elliott \& Dewaill 1995) provides some support for the hypothesis, as few large piscivorous fish species are found in European tidal marshes, in contrast to most European whole-estuary assemblages.

The value of other guild categories as tools to explore the structure and functioning of tidal marsh fish assemblages, namely vertical distribution and substratum preference guilds, was not demonstrated clearly in the present study. The spatial extent of marsh habitats may be too restricted to make the application of these guilds meaningful. Rather than attempting to explain fish vertical distribution in the tidal marsh environment using a simple 3-category vertical distribution guild, a new set of guilds based on foraging modes or predation avoidance strategies or behaviour might offer potential advantages in seeking to better understand the effects on fish behaviour of the structural complexity of tidal marsh habitats.

Further refinements are also recommended for the application of the ecological and dietary preference guilds. One shortcoming of the dietary preference categories which include invertebrates is that it is impossible to distinguish between fish taxa feeding on infaunal, epibenthic, hyperbenthic or nektonic invertebrates. Use of guilds based on foraging modes may help overcome this problem better than the division of the present dietary preference categories into a larger number of new guilds to reflect these differences. The approach adopted in the present study, and by Elliott \& Dewailly (1995), does not account for ontogenetic shifts in, for example, habitat or diet preferences. To account for such shifts, it might be necessary to assign different life stages of some species to different taxa, with guilds representing the dietary preferences of habitat preferences of each particular life stage. Species present in tidal marshes in all life stages, particularly for relatively long-lived species such as the flounder Platichthys flesus, are otherwise difficult to categorise into a single guild. Potter et al. (1993), in using similar ecological guilds to those applied here to assess the assemblage of a seasonally closed Australian estuary, identified 2 groups of estuarine resident fish species. One group comprised those species which are also represented in coastal marine waters by what are probably discrete populations, while the other consists of those species that are exclusively or almost invariably found only in estuaries.

This division is probably applicable in European estuaries, although Maitland (1974) indicated that Britain has very few truly estuarine fish species (Potter's second group). He considered that there were only 5 species in this category, the sea bass Dicentrarchus labrax, the goby Pomatoschistus microps and 3 mullet species (Chelon labrosus, Liza ramada and $L$. aurata). Although this list applied only to British estuaries, the assemblages of these areas have been shown to be similar to those of other northern European and North Sea areas, suggesting that the majority of species regarded in our study as 'estuarine resident' may fall into Potter's first category of species also found in coastal marine waters. A division of the 'estuarine resident' guild into these 2 categories would be more likely to indicate the true importance of tidal marshes for truly estuarine resident species compared to subtidal estuarine areas.

A concluding modification of the use of the functional guild concept for estuaries and tidal marshes is the need for the inclusion of the other major components of the estuarine nekton, the mobile macrocrustaceans, crabs (Portunidae), shrimps (Palaemonidae, Penaeidae, Crangonidae) and mysids being the main groups encountered in temperate estuaries and their tidal marshes (Cattrijsse et al. 1994, Kneib 1997, Mathieson \& Berry 1997). In tidal marshes, while fish species always account for the greatest part of species and family richness, nektonic crustaceans make a large, and sometimes dominant, contribution to abundance and biomass of the nektonic assemblage (Kneib 1997). Only 3 of the datasets in this study also had comparable data on the nektonic crustaceans (Forth: Mathieson unpubl. data; Westerschelde: Cattrijsse et al. 1994, Cattrijsse unpubl. data; Loire: Marchand unpubl. data) 
and, accordingly, their inclusion in this study was not possible. A fuller understanding of the importance of tidal marshes as nektonic habitats, nurseries and refugia, and as areas of great secondary productivity within estuaries, will, however, only be attained by including the full macrofaunal nektonic assemblage in future studies.

Acknowledgements. This paper is dedicated to the memory of J.M., a sadly missed and fondly remembered colleague. The authors wish to acknowledge the various opportunities that were provided for collaboration during this study by the European Commission's FAIR Concerted Action Award CT-96-1634 ('Fish in Estuaries'). A.C. acknowledges Grant BOF98/GOA/03 from the University of Gent, and Grant G2094.92 from the Belgian National Science Foundation. Data collection in the Forth estuary marsh was aided by award of a British Ecological Society Small Ecological Project Grant (No. 1149) to S.M. The authors also wish to acknowledge the efforts of the many colleagues who have contributed in large and small ways to the collection of the individual datasets included in this study. Where possible, these contributions have been acknowledged in the text. Special thanks to Kris Hostens (Westerschelde); Shona Marshall (Humber); Katharine M. Dale, Rene Swift, Sandra Telfer and Jack Cuthbert (Forth); Christian Rigaud, Brigitte Robineau and Gerard Masson (Loire); and Alberto Arias (Bay of Cadiz). Dr R. Gibson, Dr P. Maitland and Dr S. Hull provided helpful comments on an earlier version of the manuscript. For discussion of access to unpublished datasets used in the analysis, please contact S.M.

\section{LITERATURE CITED}

Allardi J, Keith P (1991) Atlas preliminaire des poissons d'eau douce de France. Museum National d'Histoire Naturelle, Paris

Allen DM, Barker DL (1990) Interannual variations in larval fish recruitment to estuarine epibenthic habitats. Mar Ecol Prog Ser 63:113-125

Aprahamian MW (1988) The biology of the twaite shad Alosa fallax (Lacepede) in the Severn estuary. J Fish Biol: 33(Suppl A):141-152

Bigelow HB, Schroeder WC (1953) Fishes of the Gulf of Maine. Fish Bull (Wash DC) 74:1-577

Blaber SJM (1991) Deep sea, estuarine and freshwater fishes: life history strategies and ecological boundaries. S Afr J Aquat Sci 17:2-11

Burd F (1989) Saltmarsh survey of Great Britain. Regional supplement No. 15, South East Scotland. Nature Conservancy Council, Peterborough

Cattrijsse A, Makwaia ES, Dankwa HR, Hamerlynck O, Hemminga M (1994) Nekton communities of an intertidal creek of a European estuarine brackish marsh. Mar Ecol Prog Ser 109:195-208

Clarke KR, Warwick RM (1994) Change in marine communities: an approach to statistical analysis and interpretation. Natural Environment Research Council, Plymouth

Costa MJ, Elliott M (1991) Fish usage and feeding in two industrialised estuaries-the Tagus, Portugal, and the Forth, Scotland. In: Elliott M, Ducrutoy JP (eds) Estuaries and coasts: spatial and temporal intercomparisons. Olsen \& Olsen, Fredensborg, p 289-297

Costa MJ, Costa JL, de Almeida PR, Assis CA (1994) Do eel grass beds and salt marsh borders act as preferential nurseries and spawning grounds for fish? An example of the Mira estuary in Portugal. Ecol Eng 3:187-195

da Sylva DP (1975) Nektonic food webs in estuaries. In: Cronin LE (ed) estuarine research, Vol 1. Academic Press, New York, p 420-447

Davis TLO (1988) Temporal changes in the fish fauna entering a tidal swamp system in tropical Australia. Environ Biol Fish 21:161-172

Deegan LA, Finn JT, Ayvazian SG, Ryder-Kieffer CA, Buonaccorsi J (1997) Development and validation of an Estuarine Biotic Integrity Index. Estuaries 20:601-617

Didier J, Kestemont P (1996) Relationships between mesohabitats, ichthyological communities and IBI metrics adapted to a European river basin (The Meuse, Belgium). Hydrobiologia 341:133-144

Drake P, Arias AM (1991a) Ichthyoplankton of a shallow coastal inlet in southwest Spain: factors contributing to colonization and retention. Estuar Coast Shelf Sci 32: 347-364

Drake P, Arias AM (1991b) Composition and seasonal fluctuations of the ichthyoplankton community in a shallow tidal channel of Cadiz Bay (SW Spain). J Fish Biol 39:245-263

Elliott M, Dewailly F (1995) The structure and components of European estuarine fish assemblages. Neth J Aquat Ecol 29(3-4):397-417

Elliott M, Taylor CJL (1989) The structure and functioning of an estuarine/marine fish community in the Forth estuary, Scotland. In: Proceedings of the 21st European Marine Biology Symposium Gdansk. Polish Academy of Sciences, Institute of Oceanology, Warsaw, p 227-240

Elliott M, O'Reilly MG, Taylor CJL (1990) The Forth Estuary: a nursery and overwintering area for North Sea fishes. Hydrobiologia 195:89-103

GESAMP (IMO/FAO/UNESCO-IOC/WMO/WHO/IAEA/UN/ UNEP Joint Group of Experts on the Scientific Aspects of Marine Environmental Protection) (1997) Marine biodiversity: patterns, threats and conservation needs. Rep Stud GESAMP 62:1-24

Gibson RN, Ezzi IA (1987) Feeding relationships of a demersal fish assemblage on the west coast of Scotland. J Fish Biol 31:55-69

Hackney CT, de la Cruz A (1981) Some notes on the macrofauna of an oligohaline tidal creek in Mississippi. Bull Mar Sci 31:658-661

Haedrich RL (1983) Estuarine fishes. In: Ketchum B (ed) Estuarine and enclosed seas. Ecosyst World 26:183-207

Halpin PM (1997) Habitat use patterns of the mummichog, Fundulus heteroclitus, in New England. I. Intramarsh variation. Estuaries 20:618-625

Hugueny B, Camara S, Samoura B, Magassouba M (1996) Applying an index of biotic integrity based on fish assemblages in a West African river. Hydrobiologia 331:71-78

Karr JR (1981) Assessment of biotic integrity using fish communities. Fisheries 6:21-27

Kneib RT (1997) The role of tidal marshes in the ecology of estuarine nekton. Oceanogr Mar Biol Annu Rev 35: $163-220$

Lever C (1997) Naturalised fishes of the world. Academic Press, London

Maitland PS (1974) The conservation of freshwater fishes in the British Isles. Biol Conserv 6:7-14

Maitland PS, Campbell RN (1992) Freshwater fishes. Harper Collins Publishers, London

Marchand J (1980) Distribution saisonnière, abondance et diversité de l'ichthyofaune de l'estuaire interne de la Loire. Ann Inst Oceanogr 56:127-137 
Marchand J, Rigaud C (1984) Etude de la faune circulante entre la Loire et les zones humides: prairie de Lavau et annexes. Rapport, CSEEL, Nantes

Marshall S, Elliott M (1996) The structure of the fish assemblage in the Humber estuary, United Kingdom. Publ Espec Inst Esp Oceanogr 21:231-242

Marshall S, Elliott M (1998) Environmental influences on the fish assemblage of the Humber estuary, UK. Estuar Coast Shelf Sci 46:175-184

Mathieson S, Berry AJ (1997) Spatial, temporal and tidal variation in crab populations in the Forth Estuary, Scotland. J Mar Biol Assoc UK 77:167-183

McDowall RM (1988) Diadromy in fishes. Croon-Helm, London

McHugh JL (1967) Estuarine nekton. In: Lauff GH (ed) Estuaries. Am Assoc Adv Sci Publ 83:581-620

McLusky DS (1989) The estuarine ecosystem. Blackie \& Son Ltd, Glasgow

Morton RM, Pollock BR, Beumer JP (1987) The occurrence and diet of fishes in a tidal inlet to a saltmarsh in southern Moreton Bay, Queensland. Aust J Ecol 12:217-237

Naismith IA, Knights B (1988) Migrations of elvers and juvenile European eels, Anguilla anguilla L, in the River Thames. J Fish Biol 33(Suppl A):161-175

Peterson GW, Turner RE (1994) The value of salt marsh edge vs interior as a habitat for fish and decapod crustaceans in a Louisiana tidal marsh. Estuaries 17:235-262

Potter IC, Beckley LE, Whitfield AK, Lenanton RCJ (1990)

Editorial responsibility: Otto Kinne (Editor),

Oldendorf/Luhe, Germany
Comparisons between the roles played by estuaries in the life cycles of fishes in temperate western Australian and southern Africa. Environ Biol Fishes 28:143-178

Potter IC, Hyndes GA, Baronie FM (1993) The fish fauna of a seasonally closed Australian estuary. Is the prevalence of estuarine-spawning species high? Mar Biol 116:19-30

Proctor J, Fraser MW, Thompson J (1982) Saltmarshes of the upper Forth Estuary. Trans Bot Soc Edinb 44:95-102

Rozas LP (1993) Nekton use of salt marshes of the southeast region of the United States. In: Magoon O et al. (eds) Proceedings of the 8th Symposium on coastal and ocean management. American Society of Coastal Engineers, New York, p 528-537

Rozas LP, Hackney CT (1984) Use of oligohaline marshes by fishes and macrofaunal crustaceans in North Carolina. Estuaries 7:213-224

Shenker JM, Dean JM (1979) The utilization of a salt marsh creek by larval and juvenile fishes: abundance, diversity and temporal variation. Estuaries 2:154-163

Wheeler A (1978) Key to the fishes of Northern Europe. Frederick Warne (Publishers) Ltd, London

Wootton RJ (1990) The ecology of teleost fishes. Chapman and Hall, London

Zimmerman RJ, Minello JT, Klima EF, Nance JM (1991) Effects of accelerated sea-level rise on coastal secondary production. In: Bolten HS, Magoon OT (eds) Coastal wetlands, Coastal Zone '91 Conference. American Society of Coastal Engineers, New York, p 110-124

Submitted: August 25, 1998; Accepted: September 20, 1999

Proofs received from author(s): September 5, 2000 